\title{
SPECTRAL ANALYSIS OF A NONLINEAR BOUNDARY-VALUE PROBLEM IN A PERFORATED DOMAIN. APPLICATIONS TO THE FRIEDRICHS INEQUALITY IN $L_{p}$
}

\author{
Yulia KoroleVA
}

Abstract. The paper deals with asymptotic expansion for $p$-Laplace boundary-value problem in a domain periodically perforated along the boundary. It is assumed that the later boundary of the domain is subject to the Neumann boundary condition while the Dirichlet condition is set on the boundary of small sets. The asymptotic expansion for the first eigenelement is constructed. This result is applied to derive the asymptotics of the best constant in the Friedrichs inequality.

Mathematics subject classification (2010): 35P30, 35B27, 34B08.

Keywords and phrases: nonlinear spectral analysis, homogenization theory, boundary-value problem with a small parameter.

\section{REFERENCES}

[1] S. Agmon, A. Douglis And L. Nirenberg, Estimates near the boundary for solutions of elliptic partial differential equations satisfying general boundary conditions, II. Comm. Pure Appl. Math. 17 (1964), 35-92.

[2] Y. Amirat, G. A. ChechKin AND R. R. GadyL'shin, Asymptotics of simple eigenvalues and eigenfunctions for the Laplace operator in a domain with oscillating boundary, Comp. Math. Math. Phys. 46 (2006), No 1, 97-110.

[3] N. S. Bakhvalov and G. P. Panasenko, Homogenization: Averaging Processes in Periodic Media, Nauka, Moscow, 1984; Kluwer, Dorderecht, 1989.

[4] A. Bensoussan, J.-L. Lions And G. Papanicolau, Asymptotic Analysis for Periodic Structures, North-Holland, Amsterdam, 1978.

[5] G. A. CHECHKIN, Asymptotic expansions of the eigenvalues and eigenfunctions of an elliptic operator in a domain with many "light" concentrated masses near the boundary. The two-dimensional case, Izv. Ross. Acad. Nauk Ser. Mat., 69 (2005), No. 4, 161-204. (English translation in Izv. Math., 69 (2005), No. 4, 805-846.)

[6] G. A. Chechin, R. R. Gadyl'shin And Yu. O. Koroleva, On the convergence of solutions and eigenelements of a boundary value problem in a domain perforated along the boundary, (English. Russian original), Differ. Equ., 46 (2010), No. 5, 667-680; translation in Differ. Uravn., 46 (2010), No. 5, 665-677.

[7] G. A. Chechioin, R. R. Gadyl'shin and Yu. O. Koroleva, On asymptotics of the first eigenvalue for Laplacian in domain perforated along the boundary, Diff. Equ., 47 (2011), No. 6, 1-10.

[8] G. A. Chechin, R. R. Gadyl'shin and Yu. O. KorolEva, On the eigenvalue of the Laplacian in a domain perforated along the boundary, (English. Russian original), Dokl. Math., 81 (2010), No. 3, 337-341; translation from Dokl. Akad. Nauk., Ross. Akad. Nauk., 432 (2010), No. 1, 7-11.

[9] G. A. Chechin, Yu. O. Koroleva, A. Meidell and L. -E. Persson, On the Friedrichs inequality in a domain perforated nonperiodically along the boundary. Homogenization procedure. Asymptotics in parabolic problems, Russ. J. Math. Phys., 16 (2009), No. 1, 1-16.

[10] G. A. Chechin, Yu. O. Koroleva, L. -E. Persson and P. Wall, O spectrum of the Lapllacian in a circle perforated along the boundary. Application to a Friedrichs-type inequality, International Journal of Differential Equations, (2011) V. 2011. Article ID 619623, 22 pages. 
[11] D. Cioranescu And P. Donato, An Introduction to Homogenization Oxford University Press, Oxford, 1999.

[12] R. R. GADYL'SHIN, Asymptotics of the minimum egenvalue for a circle with fast oscillating boundary conditions, C. R. Acad. Sci. Paris. Ser. I. 323 (1996), No. 3, 319-323.

[13] R. R. GADYL'SHIN, On the eigenvalue asymptotics for periodically clamped membranes, Algebra Anal., 10 (1998), No. 1, 3-19. (English translation in: St. Peterburg Math. J., 10 (1999), No. 1, 1-14.)

[14] A. M. IL'IN, Matching of asymptotic expansions of solutions of boundary value problems. Translated from the Russian by V. Minachin [V. V. Minakhin]. Translations of Mathematical Monographs, 102. American Mathematical Society, Providence, RI, 1992.

[15] YU. O. KoROLEVA, On a Friedrichs-type inequality in a three-dimensional domain aperiodically perforated along a part of the boundary, (English. Russian original), Russ. Math. Surv., 65 (2010), No. 4 , 788-790; translation from Usp. Mat. Nauk, 65 (2010), No. 4, 199-200.

[16] YU. O. Koroleva, The Friedrichs inequality in perforated domains. Applications to homogenization theory, in: Books of Abstract of International Scientific conference, Ufa, April 30th, 2015, (2015), $1-5$.

[17] YU. O. Koroleva, On the convergence of a nonlinear boundary-value problem in a perforated domain, International Journal of Differential Equations, 2015 (2015), Article ID 392479, 11 pages.

[18] V. MaZ'Ya, S. NAZAROV AND B. PlamenEVS KiJ, Asymptotic expansions of eigenvalues of boundary value problems for the Laplace operator in domains with small openings, Izv. Acad. Nauk SSSR, Ser. Mat., 48 (1984), 347-371.

[19] V. MaZ'Ya, S. NaZarov and B. Plamenevs KiJ, Asymptotic Theory of Elleptic Boundary Value problems in Singularly Perurbed Domains, Birkäuser, Basel, 2000, Vol.1.

[20] V. A. Marchenko And E. YA. Khruslov, Homogenized Models of Microinhomogeneous Media, Naukova Dumka, Kiev, 2005 [in Russian].

[21] V. A. Marchenko And E. Ya. Khruslov, Homogenization of Partial Differential Equations, Series: Progress in Mathematical Physics, 46 2006, XII.

[22] V. A. Marchenko and E. Ya. Khruslov, Boundary Value Problems in Domains with FineGrained Boundaries, Naukova Dumka, Kiev, 1974 [in Russian].

[23] S. A. NAZAROV, Junctions of singularly degenerating domains with different limit dimensions, I, (English. Russian original), translated from Tr. Semin. im. I. G. Petrovskogo, 314 (1995), No. 18, 3-78, J. Math. Sci., 80 (1996), No. 5, 1989-2034.

[24] S. A. NaZarov and B. A. Plamenevsky, Elliptic problems in domains with piecewise smooth boundaries. de Gruyter Expositions in Mathematics, 13. Walter de Gruyter Co., Berlin, 1994.

[25] O. A. Oleinik, A. S. Shamaev and G. A. Yosifian Mathematical Problems in Elasticity and Homogenization, Mosk. Gos. Univ., Moscow, 1990; North-Holland, Amsterdam, 1992.

[26] A. L. Pyatnits Kit, A. S. Shamaev and G. A. Chechin, Homogenization: Methods and Applications Tamara Rozhkovskaya, Novosibirsk, 2007 [in Russian].

[27] E. SÁncheZ-PALEnCia, Homogenization Techniques for Composite Media, Springer-Verlag, Berlin, 1987.

[28] I. V. SKRYPNIK, Asymptotic behaviour of solutions of nonlinear elliptic problems in punctured domains, Mat. Sb., 184 (1993), No. 10, 67-90. (English translation in: Russian Acad. Sci. Sb. Math., 80 (1993), No. 1, 191-209.)

[29] I. V. SKRYPNIK, New conditions for the averaging of nonlinear Dirichlet problems in punctured domains, Ukr. Mat. Zh., 48 (1996), No. 5, 675-694. (English translation in: Ukranian Math. J., 48 (1996), No. 5, 753-774.)

[30] I. V. SKRYPNIK AND YU. V. NAMLEEVA, Convergence of Eigenvalues and Eigenfunctions of Nonlinear Dirichlet Problems in Domains with Fine-Grain Boundary, Ukrainian Math. J., 55 (2003), No. 6, 993-1011. 